\title{
BMJ Open Association of gout and colorectal cancer in Taiwan: a nationwide population-based cohort study
}

\author{
Jen-Pin Chuang, ${ }^{1,2}$ Jenq-Chang Lee, ${ }^{3}$ Tzeng-Horng Leu, ${ }^{4,5}$ Atik Choirul Hidajah, ${ }^{6}$ \\ Ya-Hui Chang, ${ }^{7}$ Chung-Yi Li (1) 6,8,9
}

To cite: Chuang J-P, Lee J-C, Leu T-H, et al. Association of gout and colorectal cancer in Taiwan: a nationwide population-based cohort study. BMJ Open 2019;9:e028892. doi:10.1136/ bmjopen-2019-028892

- Prepublication history for this paper is available online. To view these files, please visit the journal online (http://dx.doi. org/10.1136/bmjopen-2019028892).

Received 01 January 2019 Revised 12 September 2019 Accepted 13 September 2019

Check for updates

(C) Author(s) (or their employer(s)) 2019. Re-use permitted under CC BY-NC. No commercial re-use. See rights and permissions. Published by BMJ.

For numbered affiliations see end of article.

Correspondence to

Dr Chung-Yi Li;

cyli99@mail.ncku.edu.tw

\begin{abstract}
Objectives This study aimed to determine colorectal cancer (CRC) risks among patients with gout through a follow-up study on a nationwide population-based cohort that included patients with gout and the general population in Taiwan.

Participant From the Taiwan National Health Insurance Research Database, we identified 28061 patients who were newly diagnosed with gout between 2000 and 2010 as the study cohort. We randomly selected 84248 subjects matching in gender, age and baseline year as comparison cohort. The cohorts were followed up until CRC occurrence, withdrawal from the system of National Health Insurance, or Dec. 31, 2013.
\end{abstract}

Primary and secondary outcome measures Cumulative incidences and incidence rate ratios (IRRs) of CRC between two cohorts were examined. The Cox proportional hazards model was used to evaluate risk factors associated with CRC development.

Results During the 13-year follow-up, the incidence rate of CRC development in the gout cohort reached 2.44 per 1000 person-years, which was higher than the 2.13 per 1000 person-years in the control cohort (IRR=1.15; 95\% Cl 1.04 to 1.26). After adjusting for age, gender, urbanisation status and comorbidities, including hypertension, diabetes and hyperlipidaemia, gout showed no significant association with increased risk of CRC occurrence (adjusted $\mathrm{HR}=1.03 ; 95 \% \mathrm{Cl} 0.93$ to 1.14). Conclusions Similar risks of CRC incidence were observed in patients with and without gout in Taiwan. Allopurinol and colchicine are commonly used as uratelowering drug and anti-inflammation medication in Taiwan and had been shown to reduce the risk of CRC incidence. Thus, further pharmaco-epidemiological studies should be carried out to specifically assess the role of allopurinol in the relationship between gout and CRC.

\section{INTRODUCTION}

Gout is an inflammatory disease caused by deposition of monosodium urate (MSU) crystal deposition in joints due to purine metabolism disorder. This disease is estimated to affect approximately $5 \%$ of middleaged and elderly population worldwide. ${ }^{1}$ The clinical phases of gout can be divided into 'asymptomatic hyperuricaemia', 'acute gouty arthritis' and 'chronic tophaceous gout'.

\section{Strengths and limitations of this study}

- A large number of study subjects selected from the nationwide population-based dataset strengthened the statistical power in examining associations between gout and colorectal cancer.

- The patients presented a wide range of demographic characteristics, which enabled us to perform stratified analyses according to age, sex and comorbidities.

- The National Health Insurance (NHI) database lacked data on potentially important information, such as serum uric acid level. In addition, information on colorectal cancer (CRC) stage was unavailable from the NHI.

- Our study was unable to completely adjust for known risk factors for CRC, such as smoking habits, alcohol consumption, body mass index, physical activity and family history of cancer, which are unavailable from the NHI dataset.

Hyperuricaemia is defined as serum urate levels $>8 \mathrm{mg} / \mathrm{dL}$ in men and $>7 \mathrm{mg} / \mathrm{dL}$ in women; these values are near to the supersaturated level of urate in plasma. ${ }^{3}$ Acute gouty arthritis is characterised by intense pain, swelling and stiffness of joints due to deposition of urate microcrystals. ${ }^{4}$ With time, asymptomatic periods become shorter and acute gouty occurs frequently with tophi formation, whereas chronic joint deformity may appear. If uric acid concentrations in serum are lowered below the MSU saturation point, MSU crystal deposition in tissues dissolves, and gout can be cured. ${ }^{5}$

As early as $2640 \mathrm{BC}$, gout condition was first identified by ancient Egyptians. ${ }^{6}$ Despite its long history, gout remains a major public health problem. Over the past 50 years, gout prevalence has increased; a growing number of patients suffer from this extremely painful inflammatory disease. ${ }^{7}$ The evolving evidence revealed that hyperuricaemia and gout attack are highly associated with over-intake of purine-rich food and under-excretion of uric acid. $^{58}$ 
Since 1982, cancer became the leading cause of death in Taiwan for three decades. The incidence of colorectal cancer (CRC) ranked first among all cancers and rank third in cancer mortality in Taiwan, according to the Bureau of Health Promotion Annual Report 2015. In the USA, CRC is the third most common cancer among both men and women and leading cause of cancer mortality worldwide. ${ }^{9}$ In recent years, a growing number of studies aimed to investigate the effect of gout and hyperuricaemia on carcinogenesis. ${ }^{10-14}$ Interestingly, uric acid was hypothesised to be protective in carcinogenesis due to its powerful antioxidant properties. ${ }^{10}$ Nevertheless, several studies indicated that gout is associated with increased risk of digestive system cancer, urological cancers and lung cancer. ${ }^{12} 14$

To our knowledge, metabolic syndromes, including obesity, hypertension, diabetes mellitus and dyslipidaemia, are highly associated with gout development. ${ }^{4515}$ Available evidence from epidemiological investigations and clinical studies supports the hypothesis that metabolic syndrome may be an important aetiological risk factor for the development of certain types of cancer, especially CRC..$^{16}$ Nevertheless, the precise relationship between gout and CRC development remains unclear, and the related studies are limited and mostly originate from the western countries. ${ }^{13}$ In this study, we aimed to investigate the association between gout and CRC incidence among 1 million randomly selected insured people in Taiwan, with a maximum of 13 years of follow-up.

\section{COHORT DESCRIPTION}

\section{Data sources}

In this study, we retrospectively retrieved data of the National Health Insurance Research Database (NHIRD) provided by the National Health Insurance Administration (NHIA), Ministry of Health and Welfare of Taiwan. The Longitudinal Health Insurance Database randomly selected 1 million insured people from the NHIRD by using a systematic random sampling method. NHIRD provides all inpatient and outpatient medical claims and medical orders for nearly $99 \%$ of people in Taiwan. ${ }^{18}$ To ensure privacy, all healthcare providers' identification numbers in all healthcare databases were encrypted. In Taiwan, NHIA issues major illness/injury certificates to all patients with malignant tumours. In addition, if these patients are hospitalised for diseases associated with related malignancy, they are exempted from co-payment to the National Health Insurance (NHI). The major illness/injury certificate is issued only after cancer diagnosis is pathologically confirmed. In this study, patients with CRC were identified using major illness/injury certificates for inpatients or outpatients cares to ensure accuracy of malignancy diagnosis. The data retrieved in this study excluded personal identifiers. Thus, informed consent and review by Institutional Review Board were waived.

\section{Study cohort}

Between 1 January 2000 and 31 December 2010, 41270 patients newly diagnosed with gout based on the International Classification Disease, 9th Revision, Clinical Modification (ICD-9-CM) codes 274 were identified. We excluded those with history of cancer (ICD-9-CM140-208) diagnosed before the index date (defined as the date of first diagnosis of gout) or age $<19$ years old at the index day $(n=13209)$. For each patient with gout identified in the study group, four insured participants matched with age (every 5 years), sex and index year were randomly selected from those without history of gout nor cancer as comparison cohort. Among the selected non-gout subjects $(n=112244), 27996$ non-gout subjects with prior diagnosis of all-cancer were then deleted, leaving 84248 non-gout subjects in the current analysis (figure 1).

Each patient was followed up from the index date to new diagnosis of CRC (ICD-9-CM 153-154), 31 December 2013 or withdrawal from insurance, whichever came first. Under the concern of possible unmeasured confounders, we identified baseline metabolic and anthropometric traits of metabolic syndrome, including hypertension (ICD-9-CM 401-405), diabetes mellitus (ICD-9-CM 250) and hyperlipidaemia (ICD-9-CM 272), which have been closely associated with CRC development. ${ }^{16}{ }^{17}$ Additionally, we calculated the Charlson comorbidity index (CCI) score, a weighted summary measure of clinically important concomitant diseases adopted for use with ICD-9-CM-coded administrative databases ${ }^{19}{ }^{20}$ for each study subject.

\section{Patient and public involvement}

No patient involved.

\section{Statistical analysis}

We first compared distributions of age, sex, urbanisation and comorbidities between the gout and non-gout cohorts, which were examined using $\chi^{2}$ test. We then calculated the incidence density of CRC. Gout versus non-gout incidence rate ratio (IRR) of CRC was examined. For each participant, follow-up duration was estimated from the index date to CRC diagnosis for uncensored subjects and until date of health insurance policy termination (mostly due to death) or Dec. 31, 2013 for those who were censored. We used the Cox proportional hazards model with adjustment for age, sex, urbanisation, hypertension, diabetes, hyperlipidaemia, CCI and propensity score (PS) to calculate HRs and 95\% CIs. Adjustment for age was mainly due to imbalance in average age between study groups, possibly through use of broad 5-year bands when matching. Kaplan-Meier curves were used to calculate the cumulative proportion of CRC incidence for both groups, and log-rank test was then employed to test differences between curves. We also examined the robustness of risk estimates for CRC in association with gout due to potential problem of colinearity involving simultaneous inclusion of urbanisation, comorbidity, and PS in the regression model. The results indeed showed a 


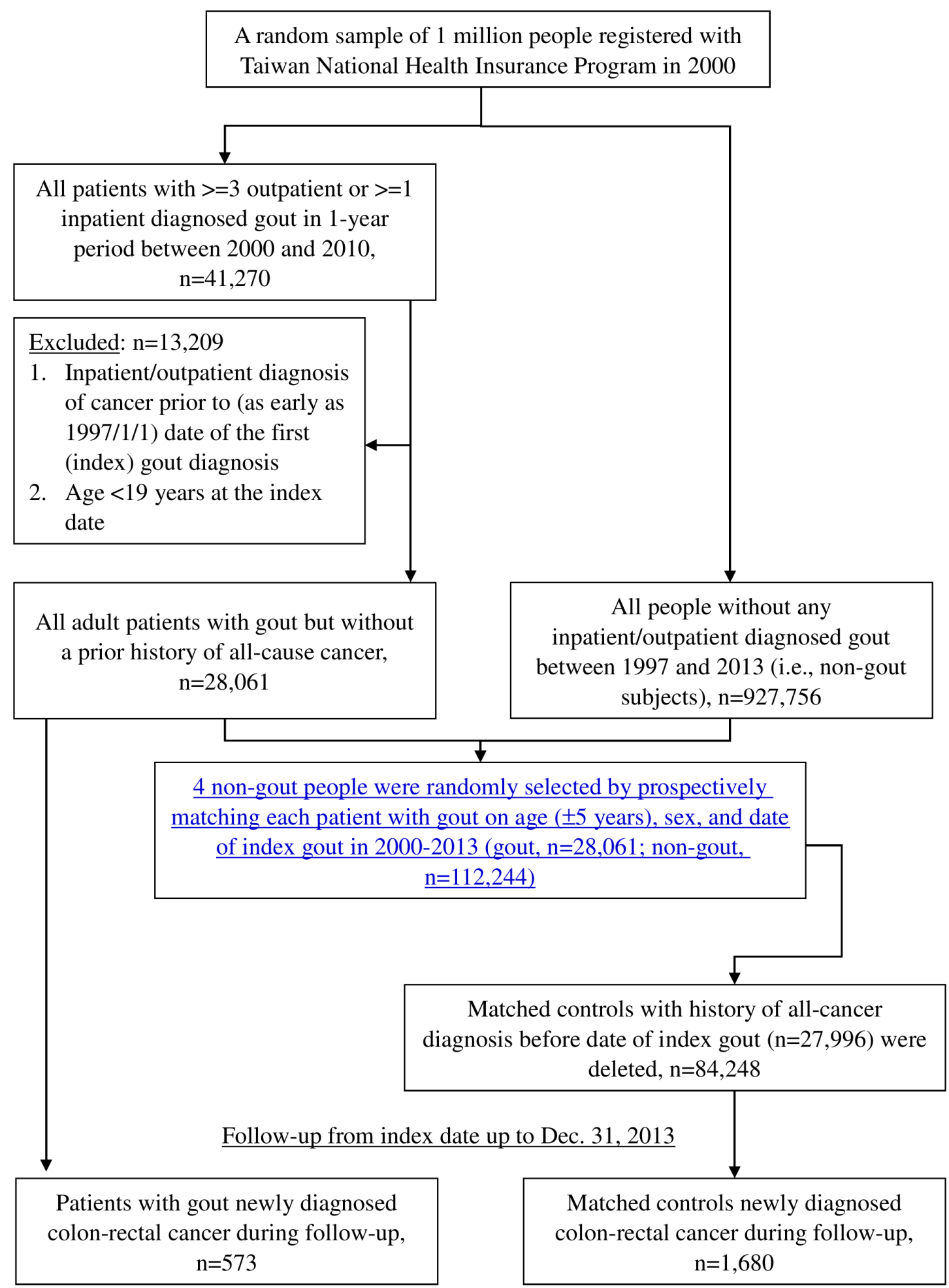

Figure 1 The flow chart of study subjects recruitment.

sign of colinearity between PS and hypertension/hyperlipidaemia, rendering a wide CI for the risk estimate associated with PS. However, such problem posed little influence of the estimation of adjusted HR of CRC in relation to gout.

Sensitivity analysis was conducted by excluding patients who developed CRC in the first 2 years after starting follow-up to assure a biologically plausible induction period. In addition, although we managed to adjust for potential risk factors for CRC, certain lifestyle factors, such as smoking, overweight and physical activities, remained unadjusted in the analysis mainly due to lack of such data in medical claims. By using all variables listed in table 1, we calculated the PS in prediction of gout diagnosis for each of the study subjects to minimise potential confounding by unmeasured confounders. We then 
Table 1 Characteristics of study cohorts

\begin{tabular}{|c|c|c|c|}
\hline & \multirow{2}{*}{$\begin{array}{l}\text { Control } \\
n=84248(\%)\end{array}$} & \multirow{2}{*}{$\begin{array}{l}\text { Gout } \\
n=28061(\%)\end{array}$} & \multirow[b]{2}{*}{$P$ value } \\
\hline & & & \\
\hline \multicolumn{4}{|l|}{ Age, years } \\
\hline$<30$ & 9775 (11.6) & $2735(9.8)$ & $<0.0001$ \\
\hline $30-44$ & $22026(26.1)$ & $6474(23.1)$ & \\
\hline $45-59$ & 24353 (28.9) & 8082 (28.8) & \\
\hline$>=60$ & $28094(33.4)$ & 10770 (38.4) & \\
\hline Mean \pm SD & $51.09 \pm 16.89$ & $53.38 \pm 17.19$ & \\
\hline \multicolumn{4}{|l|}{ Sex } \\
\hline Male & $67510(80.1)$ & 21589 (76.9) & $<0.0001$ \\
\hline \multicolumn{4}{|l|}{ Geographic area } \\
\hline Northern & 39771 (47.2) & $12749(45.4)$ & $<0.0001$ \\
\hline Central & $19735(23.4)$ & 6802 (24.2) & \\
\hline Southern & $22491(26.7)$ & 7543 (26.9) & \\
\hline Eastern & $2251(2.7)$ & $967(3.5)$ & \\
\hline \multicolumn{4}{|l|}{ Urbanisation status } \\
\hline Urban area & $36169(42.9)$ & 11079 (39.5) & $<0.0001$ \\
\hline Satellite area & 24109 (28.6) & 7949 (28.3) & \\
\hline Rural area & $23964(28.4)$ & $9030(32.2)$ & \\
\hline \multicolumn{4}{|l|}{ Years of follow-up } \\
\hline Mean \pm SD & $9.44 \pm 3.26$ & $8.43 \pm 3.48$ & 0.062 \\
\hline \multicolumn{4}{|l|}{ Comorbidity } \\
\hline Hypertension & 20367 (24.2) & $13575(48.4)$ & $<0.0001$ \\
\hline Diabetes & 10378 (12.3) & $6292(22.4)$ & $<0.0001$ \\
\hline Hyperlipidaemia & $12369(14.7)$ & $9818(35.0)$ & $<0.0001$ \\
\hline \multicolumn{4}{|l|}{$\mathrm{CCl}$} \\
\hline Mean \pm SD & $0.41 \pm 0.89$ & $0.68 \pm 1.15$ & $<0.0001$ \\
\hline \multicolumn{4}{|l|}{ PS } \\
\hline Mean \pm SD & $0.23 \pm 0.11$ & $0.31 \pm 0.14$ & $<0.0001$ \\
\hline
\end{tabular}

$\mathrm{CCl}$, Charlson comorbidity index; PS, propensity score.

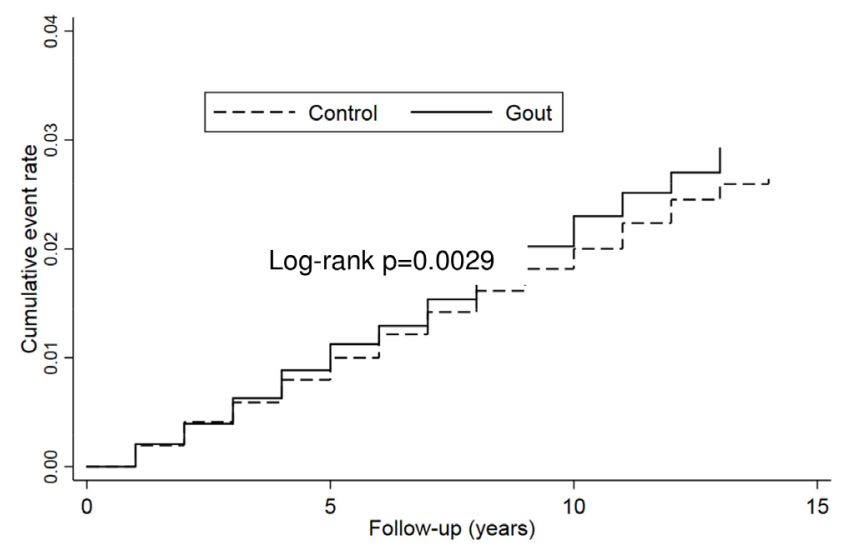

Figure 2 Kaplan-Meier analysis for colorectal cancer cumulative event rate between gout and control. conducted sensitivity analysis by treating PS and CCI score as covariates in the Cox model to assess the robustness of HR estimated from multivariate regression analysis. We performed statistical analyses with SAS (V.9.3), and a two-sided $p<0.05$ was considered statistically significant.

\section{FINDINGS TO DATE}

\section{Characteristics of study participants}

Table 1 compares the characteristics and baseline comorbidity status between the gout and non-gout cohorts. The mean $( \pm \mathrm{SD})$ age for gout and non-gout cohorts was $53.4 \pm 17.2$ and $51.1 \pm 16.9$ years, respectively. A total of $42.9 \%$ and $39.5 \%$ individuals from the non-gout and gout cohorts lived in urban areas. Mean follow-up was slightly but non-significantly $(\mathrm{p}=0.062)$ shorter in the gout group than in the non-gout group (8.4 \pm 3.5 vs $9.4 \pm 3.7$ years). The gout cohort was susceptible to comorbidities, including hypertension $(48.4 \%$ vs $24.2 \%, \mathrm{p}<0.0001)$, diabetes mellitus $(22.4 \%$ vs $12.3 \%, \mathrm{p}<0.0001)$ and hyperlipidaemia $(35.0 \%$ vs $14.7 \%, \mathrm{p}<0.0001)$.

\section{Risk and relative risk of CRC}

Kaplan-Meier survival analysis showed that CRC cumulative rate was higher in patients with gout than in non-gout controls. In addition, a significant difference in survival was observed between the two groups ( $p$ for log-rank test=0.0029) (figure 2). Table 2 compares the CRC incidence densities between the cohorts and their IRRs. A higher overall CRC incidence was observed in the gout cohort than in the non-gout cohort (2.44 vs 2.13 per 1000 person-years; IRR $=1.15,95 \%$ CI 1.04 to 1.26 ). For both gout and non-gout cohorts, the incidence density of CRC was highest in the oldest group at 4.76 versus 4.31 per 1000 person-years, respectively. The IRRs comparing the risk of gout between the gout and control groups were all elevated but non-significant for all the four age groups.

Although a higher CRC IRR (1.15) was observed in the gout cohort than in the non-gout cohort, the adverse effect of gout on CRC risk disappeared after potential confounders, including age, sex, residential location and urbanisation status, hypertension, diabetes, hyperlipidaemia, comorbidity score and PS, were considered and adjusted in the multivariate regression model. Cox's regression model indicated that compared with the non-gout cohort, the HR of CRC group presented no significant increase in patients with gout ( $\mathrm{HR}=1.04,95 \%$ CI 0.95 to 1.15 ). Once the Cox regression model additionally considered PS in addition to covariates listed in table 1, it showed HR of 1.04 (95\% CI 0.95 to 1.15 ) for the gout group (table 3 ). This finding coincided with the results estimated from the model without adjusting for PS (HR, 1.03 ; $95 \%$ CI 0.93 to 1.14 ). In the sensitivity analysis, which excluded 130 patients with gout and 334 controls who developed CRC in the first 2 years, 443 patients with gout and 1346 control patients who developed CRC were re-analysed. The covariate adjusted $\mathrm{HR}$ was estimated at 1.05 (95\% CI 0.94 to 1.17) in the reanalysis. HRs of CRC 
Table 2 IRR comparing the risk of gout between the gout and control groups

\begin{tabular}{|c|c|c|c|c|c|c|c|}
\hline & \multicolumn{3}{|l|}{ Control } & \multicolumn{3}{|l|}{ Gout } & \multirow[b]{2}{*}{ IRR (95\% Cl) } \\
\hline & $\mathbf{N}$ & Case & $\mathbf{I R}^{*}$ & $\mathbf{N}$ & Case & IR* & \\
\hline Total & 84248 & 1680 & 2.13 & 28061 & 573 & 2.44 & 1.15 (1.04 to 1.26$)$ \\
\hline$<30$ & 9775 & 23 & 0.25 & 2735 & 8 & 0.31 & 1.27 (0.57 to 2.84$)$ \\
\hline $30-44$ & 22026 & 144 & 0.67 & 6474 & 46 & 0.77 & 1.15 (0.82 to 1.60$)$ \\
\hline$>=60$ & 28094 & 1073 & 4.31 & 10770 & 375 & 4.76 & $1.10(0.98$ to 1.24$)$ \\
\hline \multicolumn{8}{|l|}{ Sex } \\
\hline Male & 67510 & 1294 & 2.05 & 21589 & 438 & 2.42 & $1.18(1.06$ to 1.31$)$ \\
\hline Female & 16738 & 386 & 2.45 & 6472 & 135 & 2.54 & 1.04 (0.86 to 1.27$)$ \\
\hline \multicolumn{8}{|l|}{ Geographic area } \\
\hline Eastern & 2251 & 38 & 1.84 & 967 & 14 & 1.92 & 1.14 (1.00 to 1.30$)$ \\
\hline \multicolumn{8}{|l|}{ Urbanisation status } \\
\hline Urban area & 36169 & 716 & 2.10 & 11079 & 229 & 2.42 & 1.15 (0.99 to 1.34$)$ \\
\hline Satellite area & 24109 & 481 & 2.14 & 7949 & 150 & 2.26 & 1.05 (0.88 to 1.27$)$ \\
\hline Rural area & 23964 & 483 & 2.17 & 9030 & 194 & 2.65 & $1.22(1.03$ to 1.44$)$ \\
\hline \multicolumn{8}{|l|}{ Comorbidities } \\
\hline Hypertension & 20367 & 679 & 3.97 & 13575 & 343 & 3.33 & $0.84(0.74$ to 0.96$)$ \\
\hline Diabetes & 10378 & 318 & 3.75 & 6292 & 162 & 3.48 & 0.93 (0.77 to 1.12$)$ \\
\hline
\end{tabular}

*Incidence rate: per 1000 patient-years.

IRR, incidence rate ratio.

in association with selected comorbidities reached 1.07 for hypertension, 0.97 for hyperlipidaemia and 1.04 for diabetes mellitus.

\section{Strengths and limitations}

Our study employed a large sample size from the nationwide population-based dataset, strengthening the statistical power for assessment of associations between gout and CRC. The patients presented a wide range of demographic characteristics, enabling us to perform stratified analyses by age, sex and comorbidities without losing precision. Nonetheless, several limitations were observed in the present study. First, the NHI database lacked data on specific potentially important information, such as serum uric acid level. Second, CRC stage information was unavailable in our database. Our study relied on claims data and diagnostic codes to ascertain patients with cancer, which were subject to disease misclassification. The potential bias arising from such potential disease misclassification would be minimal mainly because cancer diagnosis appearing in Taiwan's NHI claims should be accompanied by a major illness/injury certificate, which can be issued only after malignancy diagnosis is pathologically confirmed. Moreover, the chance of false negative is believed to be very low, similar to that of Taiwan major illness/injury certificate issued by oncologists. This certificate can be used to waive co-payment by patients for cancer treatment, which is an essential patient right. Third, we failed to completely adjust for known risk factors for CRC, such as smoking, alcohol consumption, body mass index (BMI), physical activity and family history of cancer, mainly due to unavailability of such data from NHI claims. Residual confounding could have existed and biassed the relative risk estimated in this study.

\section{DISCUSSION}

This work is by far the largest nationwide study in Taiwan to investigate the association between gout and subsequent risks of CRC. We noted that the gout group presented a 1.15-fold higher incidence of CRC than the non-gout control group in the 13-year follow-up. These findings are consistent with those of a 30-year follow-up in a Swedish study by Boffetta et al. ${ }^{13}$ However, after adjustment for age, sex and comorbid diabetes, hypertension and hyperlipidaemia, the association between gout and CRC noted 
Table 3 Relative HRs of colorectal cancer for the gout and control groups

\begin{tabular}{|c|c|c|c|c|c|}
\hline & \multicolumn{4}{|c|}{ Colorectal cancer } & \multirow[b]{3}{*}{ Adjusted HR $(95 \% \mathrm{Cl})^{\star}$} \\
\hline & \multicolumn{2}{|l|}{ No } & \multicolumn{2}{|l|}{ Yes } & \\
\hline & $n$ & $\%$ & $\mathbf{n}$ & $\%$ & \\
\hline \multicolumn{6}{|l|}{ Gout } \\
\hline No & 82568 & 98.00 & 1680 & 2.00 & 1.00 \\
\hline Yes & 27488 & 97.96 & 573 & 2.04 & 1.04 (0.95 to 1.15$)$ \\
\hline \multicolumn{6}{|l|}{ Age, years } \\
\hline$<30$ & 12479 & 99.75 & 31 & 0.25 & 1.00 \\
\hline $30-44$ & 28310 & 99.33 & 190 & 0.67 & 2.63 (1.8 to 3.86 ) \\
\hline $45-59$ & 31851 & 98.20 & 584 & 1.80 & 7.39 (5.03 to 10.87$)$ \\
\hline$\geq 60$ & 37416 & 96.27 & 1448 & 3.73 & 17.13 (11.09 to 26.46$)$ \\
\hline \multicolumn{6}{|l|}{ Sex } \\
\hline Male & 87367 & 98.06 & 1732 & 1.94 & 1.43 (1.30 to 1.59$)$ \\
\hline Female & 22689 & 97.76 & 521 & 2.24 & 1.00 \\
\hline \multicolumn{6}{|l|}{ Geographic area } \\
\hline Northern & 51349 & 97.77 & 1171 & 2.23 & 1.73 (1.27 to 2.35$)$ \\
\hline Central & 26065 & 98.22 & 472 & 1.78 & 1.23 (0.91 to 1.65$)$ \\
\hline Southern & 29476 & 98.14 & 558 & 1.86 & 1.32 (0.97 to 1.78$)$ \\
\hline Eastern & 3166 & 98.38 & 52 & 1.62 & 1.00 \\
\hline \multicolumn{6}{|l|}{ Urbanisation status } \\
\hline Urban area & 46303 & 98.00 & 945 & 2.00 & 0.95 (0.82 to 1.10$)$ \\
\hline Satellite area & 31427 & 98.03 & 631 & 1.97 & 0.92 (0.80 to 1.05$)$ \\
\hline Rural area & 32317 & 97.95 & 677 & 2.05 & 1.00 \\
\hline \multicolumn{6}{|l|}{ Comorbidity } \\
\hline Hypertension & 32920 & 96.99 & 1022 & 3.01 & 1.07 (0.66 to 1.72$)$ \\
\hline Diabetes & 16190 & 97.12 & 480 & 2.88 & 1.04 (0.92 to 1.17$)$ \\
\hline Hyperlipidaemia & 21600 & 97.35 & 587 & 2.65 & 0.97 (0.61 to 1.54$)$ \\
\hline $\mathrm{CCl}$ & & & & & $1.06(1.01$ to 1.11$)$ \\
\hline PS & & & & & $1.79(0.13$ to 24.11$)$ \\
\hline
\end{tabular}

*Adjusted model, adjusted for age, sex, urbanisation, hypertension, diabetes, hyperlipidaemia, CCI and PS.

$\mathrm{CCl}$, Charlson comorbidity index; PS, propensity score.

in our study was no longer significant. In 2012, a national population-based study from Taiwan showed that patients with gout exhibited a $15 \%$ increased risks for all-cause cancer and prostate cancer. ${ }^{21}$

In 2015, a study revealed that prevalence and incidence of gout were highly variable across various regions worldwide. The burden of gout on developed countries is usually higher than in low-income and middle-income countries. ${ }^{7}$ However, our study revealed that patients with gout are more prevalent in rural areas than the non-gout group. As reported in previous epidemiological studies, ${ }^{422}$ a strong relationship was observed between gout and metabolic syndromes, including insulin resistance, hypertension, obesity and dyslipidaemia. Such relationship was also observed in our study, in which several patients with gout suffered from high prevalence of diagnosed hypertension, diabetes and hyperlipidaemia. In the Framingham study, a high BMI has been observed in patients with gout. ${ }^{23}$ In addition to obesity, life style factors, including alcohol intake and smoking, were prevalent in the gout group. ${ }^{24-26}$ Associations between these factors and CRC have been well documented in epidemiological studies. ${ }^{27}$ However, the NHI claims data provide no information on smoking habits, alcohol consumption and BMI. Failure to adjust these risk factors may have resulted in residual confounding in our study results.

Although the antioxidant properties of uric acid play an important role in preventing the formation of carcinogenic free radicals, less evidence supports its capability to prevent cancer. ${ }^{101129}$ Chronic large bowel inflammatory conditions, such as inflammatory bowel diseases, have been considered one of the major risks of subsequent CRC development. ${ }^{30} \mathrm{~A}$ high mean number of myeloperoxidase-positive cells (neutrophils and monocytes) in normal 
mucosa has been observed in patients with CRC compared with the control group. ${ }^{31}$ Persistent inflammatory signals can continually recruit neutrophils to accumulate in the intestinal mucosa, and without efficient elimination by macrophages, the apoptotic neutrophils undergo secondary necrosis and cause pathological tissue damage by releasing detrimental intracellular contents. ${ }^{32}{ }^{33}$ Given the frequent proinflammatory cytokine production from macrophages caused by MSU stimulation, we speculate increased risk of tumour microenvironment initiation in large bowel in patients with gout.

Despite these conditions, our study indicated that the HR of CRC achieved non-significant increase in patients with gout. One possible explanation for the minimal increase in CRC risk among patients with gout is the potential protective effect of allopurinol and colchicine use for patients with gout. Allopurinol is a commonly used urate-lowering drug in Taiwan. ${ }^{34}$ A prospective randomised controlled double-blind trial study in 1993 indicated that allopurinol use (50 $\mathrm{mg}$ by mouth four times a day) prolonged survival of patients with advanced CRC..$^{35} 36$ Rajendra et al demonstrated that high dose of allopurinol $(600 \mathrm{mg} /$ day) caused significant endothelial dysfunction improvement and oxidative stress reduction in subjects with stable coronary artery disease. ${ }^{37} \mathrm{~A}$ randomised, placebo-controlled, preoperative trial demonstrated that 4-week use of allopurinol (100 or $300 \mathrm{mg}$ ) reduced oxidative activation in colon adenomatous polyps and normal adjacent tissue in 73 subjects with colorectal adenomas. ${ }^{38}$ Nonetheless, the potential protective role of allopurinol from carcinogenesis is only limited to CRC. The clinical investigation by Chen et al reported that allopurinol use is associated with increased risks of bladder cancer and all-cause cancers, especially for gout patients who had used allopurinol for more than 90 days. ${ }^{14} 39$ A recent retrospective Taiwanese study suggested that medications used by patients with gout were associated with increased risk of various cancers, including leukaemia, non-Hodgkin's lymphoma, leukaemia and breast, endometrial and cervical malignancies. ${ }^{40}$

To further explore the relationship between allopurinol and CRC risk, we conducted a posterior analysis comparing CRC risk between patients with gout with and without prescriptions for allopurinol in 1997 and 2013. Follow-up from patients with gout and taking allopurinol started from date of allopurinol prescription, whereas follow-up of those without allopurinol medication started on the date of gout diagnosis. Analytical results indicated a weak and non-significantly inverse association between allopurinol and risk of CRC among patients with gout (HR: 0.95 ; CI $95 \% 0.80$ to 1.12 ). This posterior analysis failed to entirely exclude the possibility that allopurinol could be responsible for the null association between gout and CRC, mainly because we are unable to consider a comprehensive list of risk factors for CRC in the analysis. Whether allopurinol plays an important role in preventing the development of CRC in patients with gout requires further and robust controlled studies.
Over a century, colchicine has been used for treatment and prevention of acute gouty arthritis attack due to its various mechanisms. ${ }^{414}$ Colchicine interferes with microtubule growth and dramatically abrogates the inflammatory response to urate crystal stimulation in humans. ${ }^{43}$ In addition to its anti-inflammatory effect, colchicine has been reported for its anticancer effect in vitro and in animal models. ${ }^{44} 45$ In a 12-year cohort study, colchicine use was associated with a significantly low risk of prostate cancer and CRC in male Taiwanese patients with gout. Further pharmaco-epidemiological studies should be conducted to specifically examine the relationship between colchicine use and CRC risk.

In conclusion, our study indicated that Taiwanese patients with gout diagnosis showed no association with significantly increased risk of CRC. Future pharmaco-epidemiological studies should be conducted to specifically investigate the putative link between allopurinol/colchicine and CRC risk among patients with gout. These future observational studies should thoroughly consider potential confounders in their designs. In addition, whether a dose threshold or dose-gradient effects of allopurinol exist on the risk of CRC should also be researched in future studies.

\section{Author affiliations}

${ }^{1}$ Institute of Clinical Medicine, National Cheng Kung University College of Medicine, Tainan, Taiwan

${ }^{2}$ Puzi Hospital, Ministry of Health and Welfare, Chia-Yi, Taiwan

${ }^{3}$ Department of Surgery, National Cheng Kung University College of Medicine, Tainan, Taiwan

${ }^{4}$ Institute of Basic Medical Sciences, National Cheng Kung University College of Medicine, Tainan, Taiwan

${ }^{5}$ Department of Pharmacology, National Cheng Kung University College of Medicine, Tainan, Taiwan

${ }^{6}$ Department of Epidemiology, Faculty of Public Health, Universitas Airlangga,

Surabaya, Indonesia

${ }^{7}$ Department of Public Health, National Cheng Kung University College of Medicine, Tainan, Taiwan

${ }^{8}$ Department of Public Health, College of Medicine, National Cheng Kung University, Tainan, Taiwan

${ }^{9}$ Department of Public Health, College of Public Health, China Medical University, Taichung, Taiwan

Contributors J-PC and C-YL designed the research. J-PC, J-CL, T-HL, ACH, Y-HC and $\mathrm{C}-\mathrm{YL}$ performed the research. J-PC, Y-HC and C-YL analysed the data. J-PC wrote the study, and J-CL, T-HL and C-YL critically revised the manuscript for important intellectual content.

Funding The authors have not declared a specific grant for this research from any funding agency in the public, commercial or not-for-profit sectors.

Competing interests None declared.

Patient consent for publication Not required.

Provenance and peer review Not commissioned; externally peer reviewed. Data availability statement № data are available.

Open access This is an open access article distributed in accordance with the Creative Commons Attribution Non Commercial (CC BY-NC 4.0) license, which permits others to distribute, remix, adapt, build upon this work non-commercially, and license their derivative works on different terms, provided the original work is properly cited, appropriate credit is given, any changes made indicated, and the use is non-commercial. See: http://creativecommons.org/licenses/by-nc/4.0/.

ORCID iD 
Chung-Yi Li http://orcid.org/0000-0002-0321-8908

\section{REFERENCES}

1 Mikuls TR, Farrar JT, Bilker WB. Gout epidemiology: results from the UK general practice research database, 1990-1999. Ann Rheum Dis 2005;64:267-72.

2 Chapron A, Chopin T, Esvan M, et al. Non-pharmacologic measures for gout management in the prospective GOSPEL cohort: physicians' practice and patients' compliance profiles. Joint Bone Spine 2019;86:225-31.

3 Loeb JN. The influence of temperature on the solubility of monosodium urate. Arthritis Rheum 1972;15:189-92.

4 Robinson PC, Horsburgh S. Gout: joints and beyond, epidemiology, clinical features, treatment and co-morbidities. Maturitas 2014;78:245-51.

5 Richette P, Bardin T, Gout BT. Gout. Lancet 2010;375:318-28.

6 Pillinger $\mathrm{MH}$, Rosenthal P, Abeles AM. Hyperuricemia and gout: new insights into pathogenesis and treatment. Bull NYU Hosp Jt Dis 2007:65:215-21.

7 Kuo C-F, Grainge MJ, Zhang W, et al. Global epidemiology of gout: prevalence, incidence and risk factors. Nat Rev Rheumatol 2015;11:649-62.

8 Doherty M. New insights into the epidemiology of gout. Rheumatology 2009;48(Suppl 2):ii2-8.

9 Marley AR, Nan H. Epidemiology of colorectal cancer. Int $J$ Mol Epidemiol Genet 2016;7:105-14.

10 Ames BN, Cathcart R, Schwiers E, et al. Uric acid provides an antioxidant defense in humans against oxidant- and radicalcaused aging and cancer: a hypothesis. Proc Natl Acad Sci U S A 1981;78:6858-62.

11 Strasak AM, Rapp K, Hilbe W, et al. The role of serum uric acid as an antioxidant protecting against cancer: prospective study in more than 28000 older Austrian women. Ann Oncol 2007;18:1893-7.

12 Wang W, Xu D, Wang B, et al. Increased risk of cancer in relation to gout: a review of three prospective cohort studies with 50,358 subjects. Mediators Inflamm 2015;2015:680853:6.

13 Boffetta P, Nordenvall C, Nyrén O, et al. A prospective study of gout and cancer. Eur J Cancer Prev 2009;18:127-32.

14 Chen C-J, Yen J-H, Chang S-J. Gout patients have an increased risk of developing most cancers, especially urological cancers. Scand $J$ Rheumatol 2014;43:385-90.

15 Grassi D, Ferri L, Desideri G, et al. Chronic hyperuricemia, uric acid deposit and cardiovascular risk. Curr Pharm Des 2013;19:2432-8.

16 Zhou J-R, Blackburn GL, Walker WA. Symposium introduction: metabolic syndrome and the onset of cancer. Am J Clin Nutr 2007;86:817S-9.

17 Suchanek S, Grega T, Ngo O, et al. How significant is the association between metabolic syndrome and prevalence of colorectal neoplasia? World J Gastroenterol 2016;22:8103-11.

18 JF L, Hsiao WC. Does universal health insurance make health care unaffordable? Lessons from Taiwan. Health Aff 2003;22:77-88.

19 Chen Y-C, Chang S-C, Pu C, et al. The impact of nationwide education program on clinical practice in sepsis care and mortality of severe sepsis: a population-based study in Taiwan. PLoS One 2013;8:e77414.

20 Lai T-S, Wang C-Y, Pan S-C, et al. Risk of developing severe sepsis after acute kidney injury: a population-based cohort study. Crit Care 2013;17.

21 Kuo C-F, Luo S-F, See L-C, et al. Increased risk of cancer among gout patients: a nationwide population study. Joint Bone Spine 2012;79:375-8.
22 Grassi W, De Angelis R. Clinical features of gout. Reumatismo 2012;63:238-45

23 Abbott RD, Brand FN, Kannel WB, et al. Gout and coronary heart disease: the Framingham study. J Clin Epidemiol 1988;41:237-42.

24 Choi HK, Atkinson K, Karlson EW, et al. Alcohol intake and risk of incident gout in men: a prospective study. Lancet 2004;363:1277-81.

25 Zhang Y, Woods R, Chaisson CE, et al. Alcohol consumption as a trigger of recurrent gout attacks. Am J Med 2006;119:800:e13-800.

26 Gee Teng G, Pan A, Yuan J-M, et al. Cigarette smoking and the risk of incident gout in a prospective cohort study. Arthritis Care Res 2016;68:1135-42.

27 Fagunwa IO, Loughrey MB, Coleman HG. Alcohol, smoking and the risk of premalignant and malignant colorectal neoplasms. Best Pract Res Clin Gastroenterol 2017;31:561-8.

28 Jayasekara $\mathrm{H}$, English DR, Haydon A, et al. Associations of alcohol intake, smoking, physical activity and obesity with survival following colorectal cancer diagnosis by stage, anatomic site and tumor molecular subtype. Int J Cancer 2018;142:238-50.

29 Peden DB, Hohman R, Brown ME, et al. Uric acid is a major antioxidant in human nasal airway secretions. Proc Natl Acad Sci U S A 1990;87:7638-42.

30 Mariani F, Sena P, Roncucci L. Inflammatory pathways in the early steps of colorectal cancer development. World J Gastroenterol 2014;20:9716-31.

31 Roncucci L, Mora E, Mariani F, et al. Myeloperoxidase-positive cell infiltration in colorectal carcinogenesis as indicator of colorectal cancer risk. Cancer Epidemiol Biomarkers Prev 2008;17:2291-7.

32 Ravichandran KS. Beginnings of a good apoptotic meal: the find-me and eat-me signaling pathways. Immunity 2011;35:445-55.

33 Savill J, Dransfield I, Gregory C, et al. A blast from the past: clearance of apoptotic cells regulates immune responses. Nat Rev Immunol 2002;2:965-75.

34 Lin H-C, Daimon M, Wang C-H, et al. Allopurinol, benzbromarone and risk of coronary heart disease in gout patients: a populationbased study. Int J Cardiol 2017;233:85-90.

35 Salim AS. Oxygen-Derived free-radical scavengers prolong survival in colonic cancer. Chemotherapy 1992;38:127-34.

36 Salim AS. Scavengers of oxygen-derived free radicals prolong survival in advanced colonic cancer. A new approach. Tumour Biol 1993;14:9-17.

37 Rajendra NS, Ireland S, George J, et al. Mechanistic insights into the therapeutic use of high-dose allopurinol in angina pectoris. J Am Coll Cardiol 2011;58:820-8.

38 Puntoni M, Branchi D, Argusti A, et al. A randomized, placebocontrolled, preoperative trial of allopurinol in subjects with colorectal adenoma. Cancer Prev Res 2013;6:74-81.

39 Chen C-J, Hsieh M-C, Liao W-T, et al. Allopurinol and the incidence of bladder cancer: a Taiwan national retrospective cohort study. Eur J Cancer Prev 2016;25:216-23.

40 Yang $\mathrm{H}-\mathrm{C}$, Nguyen PAA, Islam $\mathrm{M}$, et al. Gout drugs use and risk of cancer: a case-control study. Joint Bone Spine 2018;85:747-53.

41 Levy M, Spino M, Read SE. Colchicine: a state-of-the-art review. Pharmacotherapy 1991;11:196-211.

42 Cocco G, Chu DCC, Pandolfi S. Colchicine in clinical medicine. A guide for internists. Eur J Intern Med 2010;21:503-8.

43 Malawista SE, Seegmiller JE. The effect of pretreatment with colchicine on the inflammatory response to microcrystalline urate. Ann Intern Med 1965;62:648-57.

44 Lin Z-Y, Wu C-C, Chuang Y-H, et al. Anti-Cancer mechanisms of clinically acceptable colchicine concentrations on hepatocellular carcinoma. Life Sci 2013;93:323-8.

45 Craig DH, Owen CR, Conway WC, et al. Colchicine inhibits pressure-induced tumor cell implantation within surgical wounds and enhances tumor-free survival in mice. J Clin Invest 2008;118:3170-80. 